\title{
Réticulum endoplasmique : anatomie d'une membrane biologique
}

\author{
A. Amar-Costesec \\ Université de Louvain, International Institute of Cellular and Molecular Pathology et laboratoire de \\ chimie physiologique, 75, avenue Hippocrate, Bruxelles, Belgique
}

(14 $4^{e}$ réunion du groupe Développement INRA, Clermont-Ferrand, 25-27 mai 1988)

\begin{abstract}
Résumé - Le réticulum endoplasmique (RE) est un système membranaire formé de centaines de constituants protéiques et lipidiques, impliqués dans des fonctions essentielles de la physiologie cellulaire. Dans cette revue, je décris brièvement les travaux dont les résultats ont façonné nos idées sur l'organisation moléculaire du RE. L'examen au microscope électronique de coupes fines de cellules a conduit à la conception dualistique (lisse-rugueux) du RE. On a tenté, par diverses techniques, de séparer les 2 entités et de leur attribuer des fonctions distinctes. Le fait que le renouvellement des enzymes se produit suivant des modèles individuels suggère lexistence d'un RE en "mosaïque". Enfin, l'analyse en gradient de densité des microsomes de foie de rat conduit à concevoir un RE formé de 3 domaines biochimiquement distincts, où la disposition transmembranaire des protéines intégrales détermine leur distribution latérale. Je passe en revue les données actuelles sur la topographie transverse des protéines du RE et sur leur mode de biosynthèse et d'insertion dans la membrane et je confronte ces données aux prédictions du modèle.
\end{abstract}

réticulum endoplasmique - modèle — séquences topogéniques - protéines membranaires - topographie - taux de renouvellement - ribophorine

Summary - Endoplasmic reticulum : anatomy of a biological membrane. Endoplasmic reticulum (ER) is a large membranous network containing a wide variety of lipid and protein constituents which play important roles in cellular physiology. In this review, selection of experimental results are presented which have shaped our concepts of the molecular organization of ER. The morphological approach - electron microscope examination of ultra-thin sections of a variety of cells - led to the dualistic distinction between rough ER and smooth ER. Consequently, various attempts were made to separate the 2 entities and to demonstrate that they are endowed with distinct functional properties. Studies on the biogenesis of ER showed that enzymes associated with this organelle turn over independently, which was interpreted in terms of ER being biochemically organized as a mosaic. The results of isopycnic centrifugation of rat liver microsomes led us to conclude that the ER is comprised of three biochemically distinct domains and that the distribution of integral proteins in the lateral plane of the membrane (lateral topography) is primarily determined by their transmembrane disposition. Data on the transverse topography and the mode of biogenesis of ER enzymes are confronted with the predictions of this model.

endoplasmic reticulum - model - topogenic sequences - membrane protein - topography - turnover - ribophorin 


\section{INTRODUCTION}

Le réticulum endoplasmique (RE) des hépatocytes est une vaste structure membranaire, occupant plusieurs $\mathrm{m}^{2}$ par $\mathrm{g}$ de foie (Loud, 1968; Weibel et al., 1969; Wibo et al., 1971; Bolender \& Weibel, 1973; Rohr et al., 1976; Blouin et al., 1977; Bolender et al., 1978) et composée de centaines d'espèces moléculaires différentes assurant des fonctions essentielles de la physiologie cellulaire. Chacune des voies métaboliques énumérées dans le Tableau I met en œuvre plusieurs constituants : au moins 7 pour la synthèse protéique, dont un récepteur composé de 2 sous-unités (Tajima et al., 1986) et une enzyme, la peptidase du signal, comportant 7 sousunités (Evans et al., 1986); une douzaine d'enzymes pour la biosynthèse des phospholipides à choline, éthanolamine et sérine (Bell \& Coleman, 1980; Dawidowicz, 1987), une vingtaine d'enzymes pour celle du cholestérol (Chesterton, 1968; Trzaskos et al., 1982; Reinhart et al., 1987).

La coexistence sur une même membrane d'un tel nombre de protéines, impliquées dans des processus métaboliques si différents, soulève le problème de l'organisation du RE à l'échelle moléculaire. Comment les enzymes appartenant à une même voie métabolique parviennent-elles à fonctionner séquentiellement ? Nos connaissances sont encore trop fragmentaires pour qu'une réponse définitive puisse être apportée à cette question. Aussi, me contenterai-je, dans cette revue, de retracer les progrès accomplis dans la compréhension des relations entre structure et fonction au sein du RE.

\section{LISSE OU RUGUEUX : UNE CONCEP- TION DUALISTIQUE DU RE}

L'examen au microscope électronique de coupes de tissus (Palade, 1956; Palade \& Siekewitz, 1956) a conduit à une conception essentiellement dualistique du RE. Le microscope révèle en effet 2 types de structures : d'une part, des alignements parallèles de citernes aplaties portant des ribosomes (RE rugueux); d'autre part, et en continuité avec le RE rugueux, des tubules et vésicules dépourvus de ribosomes (RE lisse) (Fig. 1). Dans les hépatocytes, l'espace entre les 2 membranes du RE rugueux a une épaisseur relativement constante, ce qui lui confère une apparence rigide contrastant avec l'aspect contourné du RE lisse.

Cette remarquable dualité d'aspect suggère des différences de fonction et par conséquent de composition protéique. On pourrait à la limite supposer que RE lisse et RE rugueux sont 2 organites différents. Aussi, a-t-on cherché à mettre au point des méthodes visant à les séparer l'un de l'autre aussi complètement que possible (Rothschild, 1963; Dallner et al., 1963; revues in Dallner \& Ernster, 1968; Depierre \& Dallner, 1975). II fallut pourtant se rendre à l'évidence : sauf dans le cas de la synthèse protéique, propriété exclusive du RE rugueux, il n'est pas possible d'associer une fonction à une seule des 2 entités microsomiales, qu'il s'agisse des biosynthèses du cholestérol (Reinhart et al., 1987), de la chaîne oligosaccharidique des glycoprotéines (Ravoet et al., 1981) ou des glycérophospholipides (Bell \& Coleman, 1980; Dawidowicz, 1987). 
Tableau I. Multiplicité des fonctions du RE et liste partielle des constituants impliqués.

Biosynthèse des protéines de la membrane du RE et du réticuloplasme, des protéines sécrétoires et lysosomiales, des protéines du complexe de Golgi (?) et de la membrane plasmique

- Récepteur de la particule de reconnaissance du signal (sous-unités $\alpha$ et $\beta$ )

- Récepteur du peptide signal

- Sites de fixation des ribosomes (?)

- Sites de translocation des polypeptides

\section{Modifications co- et post-traductionnelles des protéines néo-synthétisées}

- Peptidase du signal

- Oligosaccharyltransférase

- Protéine disulfure isomérase

\section{Biosynthèse de la chaine oligosaccharidique des glycoprotéines}

- Assemblage d'un dolichol pyrophosphoryl oligosaccharide

- UDP-N-acétylglucosamine: dolichol phosphate: $\mathrm{N}$-acétylglucosamine phosphotransférase

- UDP-N-acétylglucosamine: dolichol pyrophosphoryl $\mathrm{N}$-acétylglucosamine : N-acétylglucosaminyltranférase

- GDP-mannose : dolichol phosphate: mannosyltransférase

- UDP-glucose : dolichol phosphate: glucosyltransférase

- Elagage du dolichol pyrophosphoryl oligosaccharide

- Glucosidases

- Mannosidases

\section{Biosynthèse des glycérophospholipides et des plasmalogènes}

- Enzymes des voies métaboliques du sn-glycérol 3-phosphate et de la dihydroxyacétone phosphate

- Glycérophosphate (dihydroxyacétone phosphate) acyltransférase

- Acide lysophosphatidique acyltransférase

- Diacylglycérol choline phosphotransférase

- Diacylglycérol éthanolamine phosphotransférase

- Acyl dihydroxyacétonephosphate oxydoréductase

\section{Biosynthèse du cholestérol}

- HMG CoA réductase

- Lanostérol 14-déméthylase

- Stéroïde 14-réductase

- Stéroïde 8-isomérase

\section{Biosynthèse et métabolisme des acides gras}

- NADH cytochrome $b_{5}$ réductase

- Cytochrome $b_{5}$

- Stéaroyl COA désaturase et autres désaturases

- Acide gras élongase

\section{Biosynthèse d'esters}

- Esters de CoA et d'acides gras

- Acide gras CoA ligase

- Esters de cholestérol et d'acides gras

- Acyl CoA: cholestérol acyltransférase

- Esters de rétinol et d'acides gras

- Acyl CoA: rétinol acyltranstérase 
- Esters d'acides biliaires et de taurine ou de glycine

- Acide cholique CoA ligase

Métabolisme de substances endogènes (cholestérol, stéroïdes) et des xénobiotiques

- NADPH cytochrome P-450 réductase

- Cytochrome P-450 (plus de 12 isozymes dans le foie de rat)

Glucuronoconjugaison de la bilirubine, des acides biliaires, des stéroïdes et des métabolites de xénobiotiques

- UDP-glucuronosyltransférases (au moins 4 isozymes dans le foie de rat)

- Glucosyltranférase

- Xylosyltransférase

Maintien de la giycémie

- Glucose 6-phosphate translocase

- Glucose 6-phosphatase

Conversion du cholestérol en acide cholique

- Cholestérol $7 \alpha$-hydroxylase (une forme de cytochrome P-450)

Hydratation des arènes oxydes formées par les réactions dépendant des cytochromes P-450

- Epoxyde hydrolase

Hydratation des oxydes de cholestérol et de stéroïdes formés par peroxydation

— Cholestérol 5,6 oxyde hydrolase

Dégradation oxydative de la protoporphyrine IX

- Hème oxygénase

\section{LES CONSTITUANTS PROTÉIQUES DU RE SE RENOUVELLENT INDIVIDUELLE- MENT}

Une série d'études a été consacrée à l'aspect cinétique de la synthèse des enzymes du RE : leur «turn over". Les mérites et les limitations de cette approche expérimentale ont été abondamment discutés (Bock et al., 1971; Arias et al., 1969; Kiehn \& Holland, 1970; Borgese et al., $1980)$. Les valeurs absolues des demi-vies (t 1/2) mesurées sont probablement sujettes à caution. Ces études ont néanmoins montré que les constituants protéiques du $R E$ se renouvellent à des vitesses propres à chacun : 4 j pour l'estérase (Akao \& Omura, 1972b), 2-3 j pour l'ensemble des protéines microsomiales, 3$4 \mathrm{~h}$ pour la stéaroyl CoA désaturase (Gellhorn \& Benjamin, 1966; Oshino \& Sato, 1972), I'HMG CoA réductase (Edwards \& Gould, 1972) et la cholestérol $7 \alpha$ hydroxylase (Gielen et al., 1975).

Le RE possède 2 chaînes de transport d'électrons, responsables du métabolisme des xénobiotiques ou de celui des lipides (Figs. 2 et 3). Les demi-vies des constituants de la première chaîne sont du même ordre de grandeur que celles de la moyenne des protéines du RE : $40 \mathrm{~h}$ pour l'isozyme du cytochrome P-450 inductible 


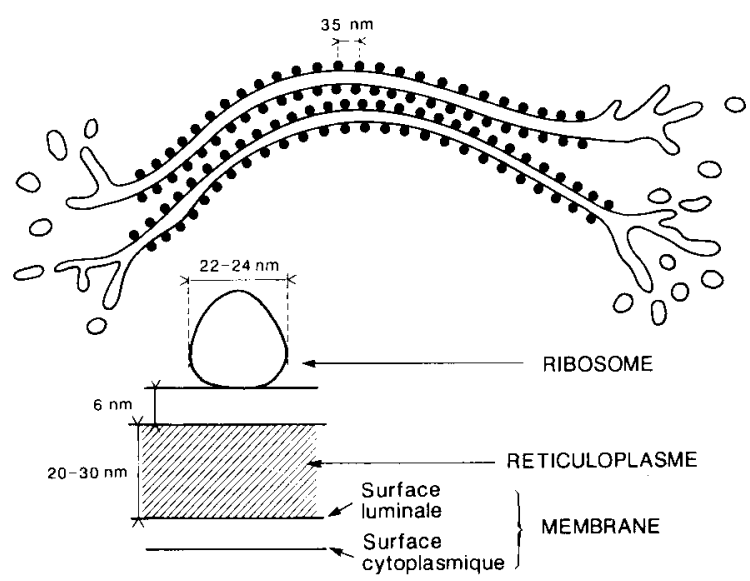

Fig. 1. Une représentation très simplifiée montrant 2 citernes du RE avec leurs portions lisses et rugueuses. Le schéma du bas donne quelques définitions et dimensions. Le plus grand diamètre du ribosome est $23,2 \pm 1,5 \mathrm{~nm}$; la distance de centre à centre entre 2 ribosomes contigus est $34,5 \pm 3,6 \mathrm{~nm}$ (Ojakian et al., 1977).

par le phénobarbital (Fujii-Kuriyama et al., 1978), $70 \mathrm{~h}$ pour la NADPH cytochrome P450 réductase (Kuriyama et al., 1969). Par contre, ceux de la seconde chaîne - à l'exception de la stéaroyl CoA désaturase, déjà citée - ont des demi-vies plus longues : $6 \mathrm{j}$ (NADH cytochrome 65 réductase) (Okada \& Omura, 1978) et $4 \mathrm{j}$ (cytochrome b5 (Kuriyama et al., 1969).

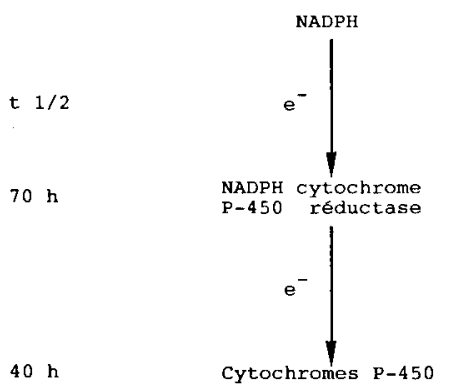

Fig. 2. Demi-vie des protéines de la voie des mono-oxygénases. Les accepteurs et les connexions avec l'autre chaîne microsomiale de transport d'électrons ne sont pas représentés. La stoechiométrie des réactions n'est pas indiquée.
A la suite de ces observations, le RE est apparu comme a mosaic in which each patch or tessera represents a specific group of enzymes and in which, accordingly, all tesserae are not biochemically equivalent to one another (Omura et al., 1967).

De nombreuses tentatives ont été faites pour établir expérimentalement la microhétérogénéité du RE, en particulier l'organi-

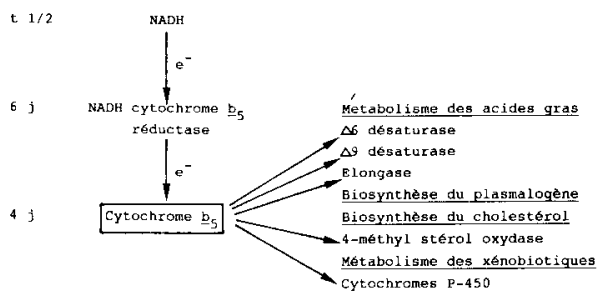

Fig. 3. Demi-vie des protéines de la chaîne microsomiale de transport d'électrons fonctionnant à partir du NADH cytosolique. Les enzymes de cette chaîne sont responsables de la synthèse des acides gras polyinsaturés. 
sation des chaînes de transport d'électrons microsomiales en complexes multimoléculaires (revue in Yang, 1977). On a essayé de fragmenter le RE à la taille des complexes et de séparer les fragments par centrifugation (revue in Depierre \& Dallner, 1975), immunoprécipitation et immunoadsorption (Kawajiri et al., 1977). On a également tenté d'observer les complexes au microscope électronique en utilisant des anticorps marqués à la ferritine (Morimoto et al., 1976; Matsuura et al., 1978, 1979). Ces travaux n'ont pas conduit aux conclusions espérées. La tendance actuelle est de considérer que les oxydo-réductases ne sont pas organisées en complexes et que les cytochromes, en particulier, jouissent d'une assez grande liberté de mouvement dans le plan latéral de la membrane (Estabrook et al., 1981).

\section{RE ET MICROSOMES}

Une difficulté dans l'étude du RE est due au fait que l'organite isolé à partir d'homogénats tissulaires a perdu sa structure en citernes. En effet, l'homogénéisation du tissu provoque la vésiculation du RE sans, cependant, entraîner de modification rédhibitoire de son architecture. Les polysomes sont fixés à la face externe des vésicules rugueuses (correspondant à la face cytoplasmique du RE rugueux). Les protéines du réticuloplasme se retrouvent à l'intérieur des vésicules. Après cryofracture, la densité et la répartition des particules entre faces $P$ et $E$ sont les mêmes dans les microsomes isolés que dans le RE in situ (Losa et al., 1978).

Cela traduit bien l'extrême plasticité de cet organite dont une des fonctions essentielles est précisément de donner naissance à des vésicules. Le RE a d'ailleurs été isolé sous forme de microsomes (Claude, 1938) avant même d'avoir été identifié dans les cellules. Les vésicules microsomiales ont un diamètre moyen de 140 nm (Wibo et al., 1971).

\section{L'ANALYSE DES MICROSOMES PAR CENTRIFUGATION ISOPYCNIQUE}

La centrifugation en gradient de densité a tenu une grande place dans l'étude biochimique du RE; pendant près de 2 décennies, on a cru possible de séparer les vésicules lisses des vésicules rugueuses. La centrifugation des microsomes en gradient analytique (Amar-Costesec et al., 1969; Beaufay et al., 1974) montre pourquoi cet espoir est vain. La densité d'équilibre des vésicules microsomiales - notamment celle des vésicules rugueuses - a un caractère polydispersé marqué : les distributions s'étalent sur toute la hauteur du gradient (Fig. 4).

Les constituants du RE présentent 3 types de distribution de densité (Fig. 4 et Tableau II). Les enzymes dites du groupe b se distribuent comme la NADPH cytochrome P-450 réductase : elles forment un pic à la densité 1,14, qui est la densité modale des vésicules lisses. Les enzymes du groupe $c$ se distribuent comme la glucose 6-phosphatase et forment 2 épaulements aux densités 1,14 et 1,23 . Enfin, les constituants du groupe d, en particulier l'ARN et les ribosomes, ont une distribution de densité symétrique par rapport à celle de la flavoprotéine, avec un pic à 1,23.

Au cours d'expériences associant analyses biochimique et stéréologique, Wibo et al. (1971) ont montré que le caractère polydispersé de la densité d'équilibre des microsomes est en relation avec la charge en ribosomes des vésicules (Fig. 5). En 


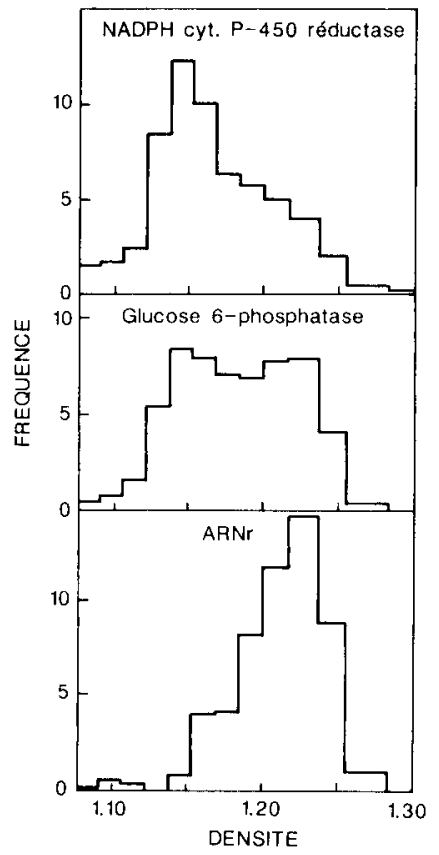

Fig. 4. Distributions de densité des constituants du RE. Une fraction subcellulaire, contenant l'ensemble des organites subcellulaires, à l'exception des noyaux (granules cytoplasmiques) a été centrifugée à travers un gradient linéaire de saccharose- $\mathrm{H}_{2} \mathrm{O}$. L'équilibre isopycnique est atteint après $140 \mathrm{~min}$ de centrifugation à $40000 \mathrm{rpm}$ dans le rotor $E-40$. On recueille 15 sous-fractions dont on mesure la densité et les propriétés biochimiques. La distribution de densité de l'ARNr est essentiellement celle des ribosomes. Les caractéristiques du rotor E-40 ainsi que le mode de représentation des résultats sont décrits dans Beaufay \& Amar-Costesec (1976). D'après AmarCostesec \& Beaufay (1981).

d'autres termes, la position d'une vésicule au sein du gradient est essentiellement déterminée par le nombre de ribosomes qu'elle porte par unité de surface.

En se basant sur les données morphologiques, on s'attendrait à ce que le RE rugueux donne naissance à une population homogène de vésicules rugueuses. On pourrait donc conclure que les vésicules ayant une charge intermédiaire en ribosomes (celles qui s'équilibrent entre le pic de densité 1,14 et celui de densité 1,23 ) sont le résultat d'une dégranulation partielle au cours de l'homogénéisation.

Nous avons au moins deux raisons de penser qu'il n'en est rien. Bien que notre milieu d'homogénéisation ne contienne pas de $\mathrm{Mg}^{2+}$ dont l'effet stabilisateur sur la jonction ribosome-membrane est bien établi, nous trouvons qu'après centrifugation différentielle du'un homogénat, la répartition de I'ARN entre fractions nucléaire, mitochondriale, microsomiale et cytosolique est comparable à celle obtenue avec un milieu renfermant du $\mathrm{Mg}^{2+}$ (Blobel \& Potter,



Fig. 5. La position d'une vésicule au sein du gradient est essentiellement déterminée par sa charge en ribosomes. Des microsomes ont été centrifugés à l'équilibre dans les conditions décrites dans la légende de la Fig. 4 pour les granules cytoplasmiques. Dans les 15 sous-fractions obtenues, on a compté les ribosomes par stéréologie (graphique du haut, Wibo et al., 1971) et on a mesuré la teneur en ARNr. Le pic d'ARNr est à la densité 1,21 au lieu de 1,23 dans la Fig. 4 car les granules cytoplasmiques contiennent une population de vésicules très rugueuses qui, lorsqu'on prépare les microsomes, est écartée avec la fraction mitochondriale. 
Tableau II. Classification en groupes des constituants du RE en fonction de leur distribution en gradient de densité.

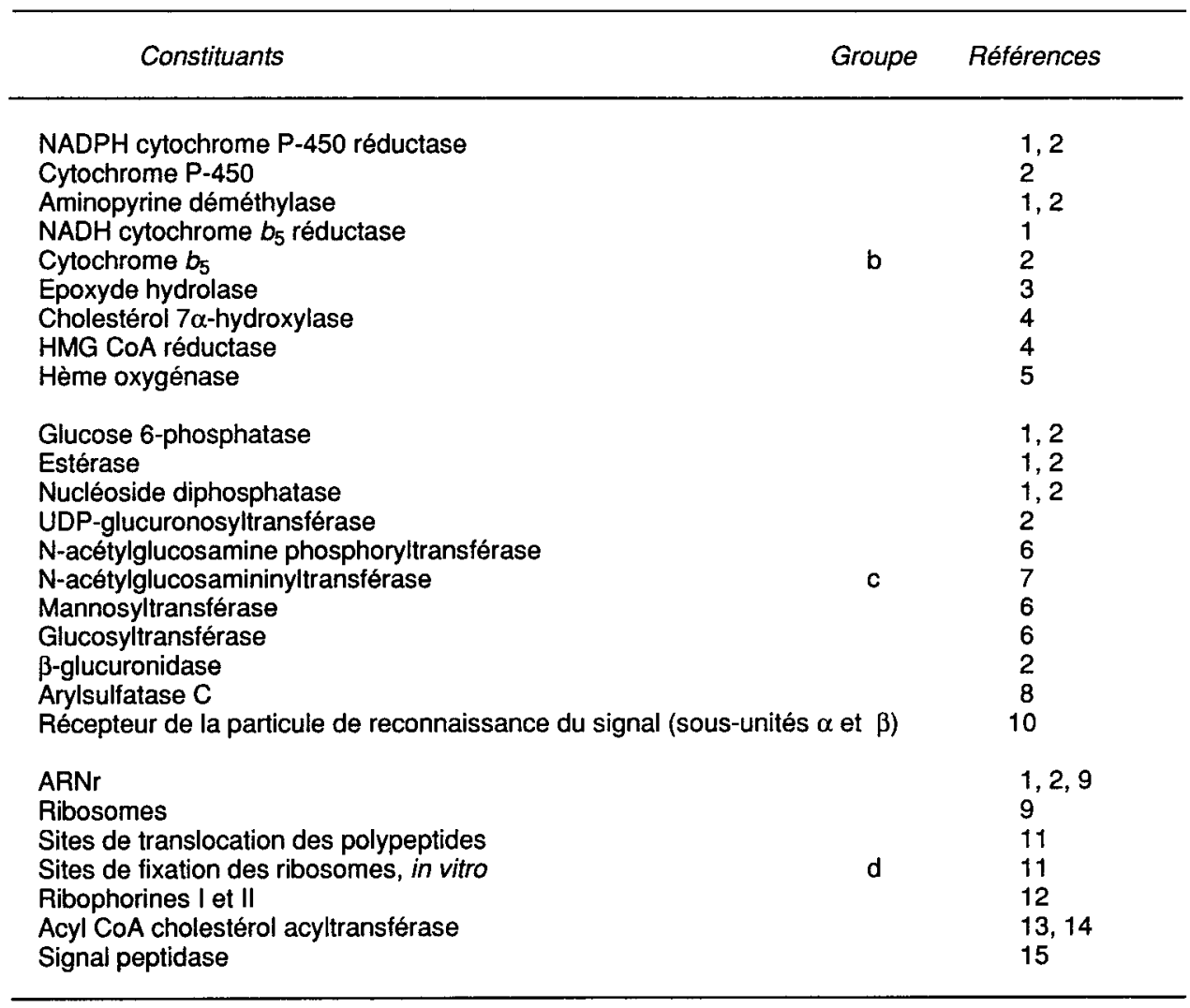

(1) Amar-Costesec et al., 1969; (2) Beaufay et al., 1974; (3) Amar-Costesec (résultats non publiés); (4) Mitropoulos et al., 1978; (5) Brunyeel, Beaufay \& Amar-Costesec (résultats non publiés); (6) Ravoet et al., 1981; (7) Van Den Eynde et al., 1985; (8) Guimard \& Amar-Costesec (résultats non publiés); (9) Wibo et al., 1971; (10) Lauffer et al., 1985; (11) Amar-Costesec et al., 1984; (12) Marcantonio et al., 1984; (13) Venkatesan et al., 1980; (14) Soni \& Amar-Costesec (résultats non publiés); (15) Amar-Costesec et al., 1989.

1967). D'autre part, on peut, après détachement des ribosomes par lavage des microsomes avec des solutions d'agents chélateurs, fixer une quantité de $\left({ }^{3} \mathrm{H}\right)$ ribosomes correspondant à $107 \mu \mathrm{g}$ $\mathrm{d}^{\prime} \mathrm{ARNr} / \mathrm{mg}$ de protéine, sur des sites à haute affinité $\left(\mathrm{Ka}=10^{8} \mathrm{M}^{-1}\right)$. Cette quantité est équivalente à celle qui se trouvait sur les membranes avant dégranulation (97 $\mu \mathrm{g}$ d'ARNr/mg de protéine) (Amar-
Costesec et al., 1984). Le nombre de sites libérés au cours de l'homogénéisation du foie et du fractionnement semble donc modeste.

La position d'une vésicule au sein du gradient étant déterminée en premier lieu par sa charge en ribosomes, la distribution de densité de l'ARN reflète celle des ribosomes dans le plan latéral de la mem- 
brane. II en va de même pour les autres constituants du RE, et les expériences de centrifugation en gradient de densité nous apprennent que les constituants $b, c$ et $d$ se répartissent inégalement entre les portions lisse et rugueuse du RE.

\section{LA DISTRIBUTION LATÉRALE DES CONSTITUANTS DU RE EST DÉTERMI- NÉE PAR LEUR MODE D'INSERTION TRANSMEMBRANAIRE}

On peut faire apparaître une corrélation entre la répartition des enzymes dans le plan de la membrane (topographie latérale), telle que nous la déduisons des données de la centrifugation isopycnique, et leur mode d'insertion transmembranaire (topographie transversale) (Tableau III). Si l'on fait provisoirement abstraction des protéines d, cette corrélation peut s'énoncer de la manière suivante : les protéines dont la chaîne polypeptidique est largement exposée du côté cytoplasmique de la membrane ont une distribution latérale de type b; les enzymes du réticuloplasme, ou ceux dont la chaîne polypeptidique est orientée du côté luminal, ont une distribution de type $c$. Afin de rendre cette corrélation plus

Tableau III. Relation entre la disposition transmembranaire des protéines du RE et leur appartenance aux groupes b et $c$.



* sauf mention contraire, il s'agit de protéines de foie de rat; ${ }^{1}$ foie de bcuf; ${ }^{2}$ cellules d'ovaire de hamster chinois.

** Colonne de gauche : rapport du nombre de résidus d'acides aminés émergeant de la membrane du cóté cytosolique au nombre total de résidus; colonne de droite : ce rapport est exprimé en pourcentage.

(1) Falany et al., 1987; (2) Porter et al., 1986; (3) Fujii-Kuriyama et al., 1982; (4) Gonzales et al., 1985; (5) Porter \& Kasper, 1985; (6) Ozols et al., 1985; (9) Mackenzie, 1986a; (10) lyanagi et al., 1986; (11) Mackenzie, 1986b; (12) Mackenzie, 1987; (13) Akao \& Omura, 1972a; (14) Robbi \& Beaufay, 1983; (15) Kuriyama, 1972. 
perceptible, j'ai, dans le Tableau III, exprimé en pourcentage le nombre de résidus d'acides aminés "cytoplasmiques" par rapport au nombre total de résidus. Ces nombres sont obtenus à partir de la séquence primaire et de l'indice d'hydropathie de polypeptide, qui permet de situer la position du segment d'ancrage dans la membrane. De plus, la topographie transmembranaire de la plupart des enzymes figurant dans le Tableau II a été établie par diverses approches expérimentales : tests de latence structurale en présence de détergents, attaque protéolytique ménagée de vésicules microsomiales intactes, action de réactifs non perméants, examen au microscope électronique de vésicules microsomiales après réaction avec des anticorps marqués à la ferritine.

\section{UN MODĖLE DÉCRIVANT L'ARRANGE- MENT DES PROTÉINES DU RE}

La corrélation entre topographies latérale et transmembranaire m'a suggéré un modèle d'organisation de la membrane du RE (Amar-Costesec, 1978a et $b$; revues générales in Amar-Costesec, 1979; AmarCostesec \& Beaufay, 1981; Beaufay et al., 1981) dans lequel les enzymes de la face luminale (groupe $\mathrm{c}$ ) sont réparties de manière à peu près uniforme dans le plan latéral de la membrane. Au contraire, il existerait une restriction à la mobilité latérale des enzymes de la face cytoplasmique (groupe b). Le pic formé à la densité 1,14 par les enzymes b étant symétrique de celui formé à la densité 1,23 par l'ARN, cette restriction serait en rapport avec la présence des ribosomes : leur jonction à la membrane ferait obstacle à la diffusion des protéines $b$. Mais le ribosome ne peut pas, à lui seul, constituer l'obstacle, car sa plus grande dimension est d'environ
$23 \mathrm{~nm}$ (Fig. 1), alors que le domaine $d$ "gèle" une surface de membrane correspondant à un cercle de 52 à $57 \mathrm{~nm}$ de diamètre par ribosome (Amar-Costesec \& Beaufay, 1981). L'obstacle à la diffusion des protéines $b$ reflète l'existence d'agrégats de protéines membranaires (protéines d) ou d'un changement de phase des phospholipides de la bi-couche.

Selon ce modèle, le RE rugueux serait une structure fortement dissymétrique, avec une face luminale où les enzymes,

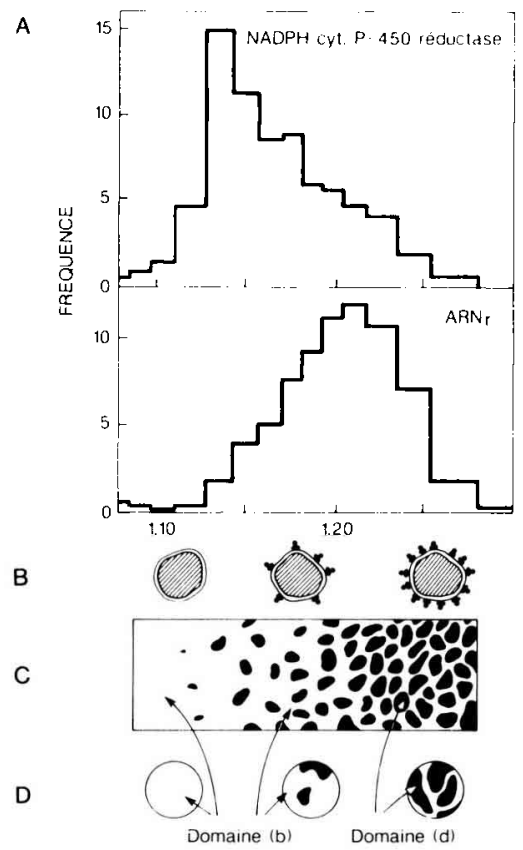

Fig. 6. Organisation des domaines $b$ et $d$ a la surface cytoplasmique du RE. (A) Distributions de densité de la flavoprotéine et de l'ARNr. (B) Les vésicules microsomiales s'alignent le long du gradient de densité en fonction de leur charge en ribosomes par unité de surface. (C) Arrangement des protéines membranaires de la surface cytoplasmique du RE, déduit des distributions montrées en (A). Les protéines $d$ forment des agrégats entre lesquels les protéines $b$ se déplacent latéralement. (D) La surface des vésicules microsomiales est supérieure à la surface d'un agrégat de protéines $d$. 
sous forme d'entités moléculaires individuelles ou de complexes, pourraient diffuser sans rencontrer d'obstacles majeurs et une face cytoplasmique ou les protéines b ne pourraient se déplacer qu'entre les agrégats de protéines d (Fig. 6).

\section{IDENTIFIER LES PROTÉINES D}

La découverte de protéines $d$ a étayé le modèle proposé. Rappelons que dans notre système expérimental, un constituant d se caractérise par une distribution en gradient de densité superposable à celle de l'ARN (Fig. 4). La présence des ribosomes indique qu'une fonction essentielle du domaine $d$ - sinon la seule - est la synthèse de certaines classes de protéines (Tableau I). Le transfert de la chaîne oligosaccharidique des glycoprotéines étant un événement cotraductionnel, nous avons d'abord pensé aux transférases de la voie du dolichol phosphate. Molnar et al. (1969) avaient observé que la mannosyltransférase ne se trouve que dans les microsomes rugueux. Nos résultats ont montré que les enzymes qui synthétisent les dérivés monoglycosylés du dolichol phosphate par transfert de mannose, glucose, $\mathrm{N}$-acétylglucosamine phosphate (Ravoet et al., 1981) ou Nacétylglucosamine (Van den Eynde et al., 1985) sont présentes aussi bien dans les portions lisses que dans les portions rugueuses du RE. A quelques réserves près (voir la discussion in Ravoet et al., 1981), les transférases que nous avons étudiées se classent parmi les constituants $c$.

En revanche, les sites à haute affinité fixant les ribosomes in vitro en l'absence de synthèse protéique, les sites de translocation à travers la membrane du RE des polypeptides synthétisés in vitro (Amar-
Costesec et al., 1984), les ribophorines I et II (Marcantonio et al., 1984) et la peptidase du signal (Amar-Costesec et al., 1989) présentent des distributions de type d (Tableau IV).

Les constituants que nous venons d'énumérer, à l'exception des ribophorines (Kreibich et al., 1978), sont tous impliqués dans la synthèse protéique. Bien que la fonction des ribophorines reste à établir, le fait qu'il en existerait un hétérodimère par ribosome (Marcantonio et al., 1984) suggère un rôle dans la jonction ribosomemembrane. II est surprenant de constater que les sous-unités $\alpha$ et $\beta$ du récepteur de la particule de reconnaissance du signal, qui jouent un rôle important dans l'adressage des polysomes au RE (Meyer \& Dobberstein, 1980; Gilmore et al., 1982), ne présentent pas de distribution de type $d$ (Tajima et al., 1986). En revanche, à la suite de Venkatesan et al. (1980), nous avons montré que l'acyl CoA:cholestérol acyltransférase, une enzyme catalysant la formation d'esters de cholestérol et d'acides gras, possède une distribution de densité de type d (Soni \& Amar-Costesec, résultats non publiés), ce qui indique que les constituants $d$ n'ont pas pour fonction exclusive la synthèse protéique.

\section{L'ASYMÉTRIE S'ÉTABLIT AU MOMENT DE LA SYNTHĖSE DES PROTÉINES DU RE}

J'ai résumé dans le Tableau IV l'essentiel de nos connaissances sur la biosynthèse des protéines du RE. Celles-ci sont synthétisées selon 3 modes différents.

Les protéines de la chaîne de transport d'électrons transitant par le cytochrome $b_{5}$ constituent un cas particulier : synthèse par des polysomes cytosoliques libres, in- 
sertion post-traductionnelle par une séquence se trouvant à l'extrémité carboxyterminale du polypeptide. Ce mode de synthèse par les polysomes libres s'apparente à celui des protéines nucléaires, mitochondriales, chloroplastiques, péroxysomiales et cytosoliques. L'extrémité carboxy-terminale de ces 3 protéines $b$ constitue-t-elle un signal d'adressage au RE?

Les autres protéines du RE sont synthétisées par des polysomes liés aux membranes rugueuses mais selon 2 modes différents. La séquence topogénique des protéines $b$ est un peptide signal amino-terminal qui sert de séquence d'insertion cotraductionnelle; l'essentiel du polypeptide reste du côté cytoplasmique de la membrane.

Les protéines c sont synthétisées avec une séquence signal amino-terminale transitoire; la translocation cotraductionnelle intéresse la plus grande partie du polypeptide, la séquence d'insertion transmembranaire (stop transfert) se trouvant vers l'extrémité carboxy-terminale. Autre différence entre protéines $b$ et $c$ : seules ces dernières sont glycosylées, ce qui est compatible avec la localisation du site actif de l'oligosaccharyltransférase du côté luminal de la membrane (revue in Beaufay et al., 1985); seuls les polypeptides exposant la séquence consensus $\mathrm{N}-\mathrm{X}-\mathrm{S} / \mathrm{T}$ du côté luminal peuvent être glycosylés.

Comment se situent les protéines d dans ces comparaisons ? Nos connaissances se limitent aux seules ribophorines I et II dont la séquence primaire (Crimaudo et al., 1987; Harnik-Ort et al., 1987) et le mode de biosynthèse (Rosenfeld et al., 1984) ont été établis. En fait, elles présentent l'ensemble des caractéristiques des protéines c. La seule différence notable est que les ribophorines ont un domaine cytoplasmique plus important que celui des glucuronosyltransférases : 150 rési- dus pour la ribophorine 1 de foie de rat (Harnik-Ort et al., 1987), 70 pour la ribophorine II de foie humain (Crimaudo et al., 1987) et seulement 19-20 pour les UDP. glucuronosyltransférases, mais la disposition transmembranaire est très semblable.

Comment expliquer que des protéines dont le mode de biosynthèse et d'insertion sont aussi proches connaissent des destinations différentes, les unes (UDP-glucuronosyltransférases) diffusant dans toute la membrane alors que les autres restent confinées dans le domaine $d$ ? Comment, d'une manière plus générale, expliquer que certaines protéines (NADPH cytochrome $P-450$ réductase, cytochromes $P$ 450 , UDP-glucuronosyltrans-férases) puissent quitter le domaine $\mathrm{d}$ où elles ont été synthétisées?

D'un point de vue biosynthétique, les protéines du RE se classent donc en 4 catégories :

- le cytochrome $b_{5}$ et les oxydoréductases associées;

- le cytochrome P-450, sa réductase et l'époxyde hydrolase;

- les enzymes périphériques, résidentes permanentes du réticuloplasme;

- les protéines intégrales des domaines $\mathrm{c}$ et d. Cette observation, loin de remettre en cause nos conceptions sur l'organisation du RE en 3 domaines biochimiquement distincts, est une incitation à poursuivre l'étude des séquences topogéniques des protéines de cet organite.

\section{CONCLUSION}

Au cours des dernières décades, les progrès dans la compréhension de l'organisation des membranes sont venus de l'étude 
Réticulum endoplasmique

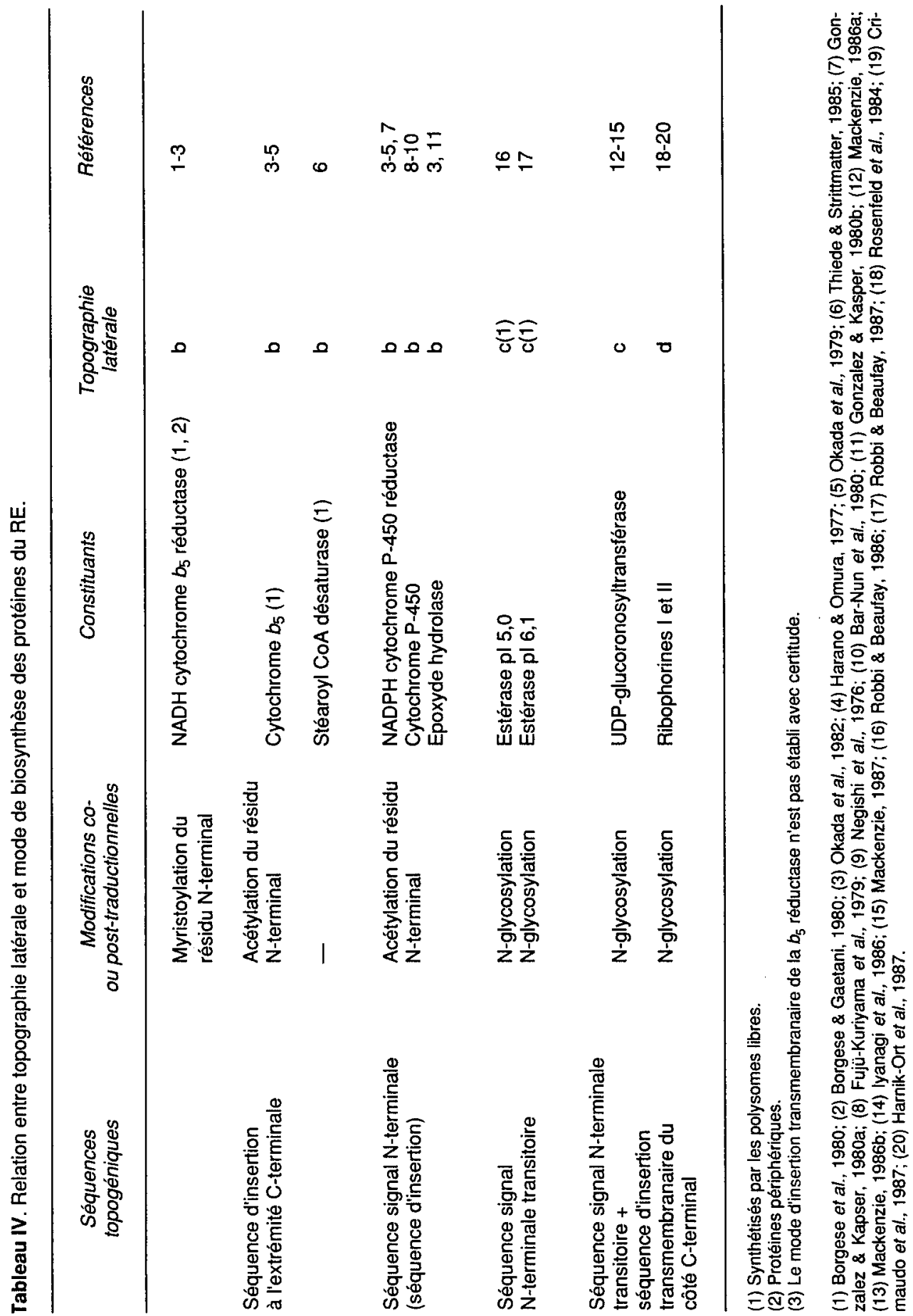


multidisciplinaire des structures les plus simples, comme la membrane des globules rouges. L'étude du RE avec ses centaines de constituants protéiques et lipidiques différents est une entreprise de très longue haleine. En revanche, le fait que certaines voies métaboliques - synthèse protéique, métabolisme des xénobiotiques, synthèse des phospholipides - fassent l'objet d'investigations poussées est un avantage appréciable pour une meilleure connaissance de cet organite. L'importance fonctionnelle du RE justifie les efforts consentis pour l'étudier. Nos connaissances portent surtout sur les domaines $b$ et $c$. Nous avons identifié, par électrophorèse bidimensionnelle à haute résolution, une vingtaine de polypeptides susceptibles d'appartenir au domaine d (Arroyo, Willard-Gallo, Beaufay \& AmarCostesec, résultats non publiés). Il reste à en définir la fonction, la disposition transmembranaire et à découvrir la nature des interactions responsables de la création de domaines au sein d'une matrice membranaire fluide.

\section{REMERCIEMENTS}

J'exprime ma gratitude au $\mathrm{Dr} \mathrm{H}$. Beaufay qui a fait de nombreuses suggestions pour améliorer le contenu du manuscrit et à Mlle M. GarbazKaufmann pour son travail d'édition. Les travaux sur les microsomes ont été financés par la Programmation de la Politique scientifique, Service du Premier Ministre de l'État belge (pôles d'attraction interuniversitaires, convention $n^{\circ} 7$ et actions de recherche concertées, conventions $n^{\circ} 82 / 87-39$ et $n^{\circ} 88 / 93-122$ ), par le Fonds de la Recherche Fondamentale Collective (conventions nos $2.4540 .80,2.4540 .84$ et 2.4549 .88 ) et par le Fonds National de la Recherche Scientifique (convention $n^{\circ}$ 1.5.182.85F).

\section{RÉFÉRENCES}

Akao T. \& Omura T. (1972a) Acetanilidehydrolyzing esterase of rat liver microsomes. I. Solubilization, purification and intramicrosomal localization. J. Biochem. 72, 1245-1256

Akao T. \& Omura T. (1972b) Acetanilidehydrolyzing esterase of rat liver microsomes. II. Turnover studies. J. Biochem. 72, 1257-1259

Amar-Costesec A. (1978a) The molecular organization of endoplasmic reticulum in liver : a plausible model to account for the enzymic heterogeneity. Arch. Internat. Physiol. Biochim. 86, 193-194

Amar-Costesec A. (1978b) The distribution of enzymes in the plane of endoplasmic reticulum membranes of rat liver. Arch. Internat. Physiol. Biochim. 86, 843-844

Amar-Costesec A. (1979) Hétérogénéité enzymatique du réticulum endoplasmique. In : Mises au Point de Biochimie Pharmacologique (Siest G. \& Heusghem C., eds) $2^{\mathbf{B}}$ série, Masson, Paris, $94-122$

Amar-Costesec A. \& Beaufay H. (1981) A structural basis of enzymic heterogeneity within liver endoplasmic reticulum. J. Theor. Biol. 89, 217 . 230

Amar-Costesec A., Beaufay H., Feytmans E., Thines-Sempoux D. \& Berthet J. (1969) Subfractionation of rat liver microsomes. In : Microsomes and Drug Oxidations (Gillette J.R., Conney A.H., Cosmides G.J., Estabrook R.W. \& Fouts, J.R., eds). Academic Press, New York, 41-58

Amar-Costesec A., Todd J.A. \& Kreibich G. (1984) Segregation of the polypeptide translocation apparatus to regions of the endoplasmic reticulum containing ribophorins and ribosomes. I. Functional tests on rat liver microsomal subfractions. J. Cell Biol. 99, 2247-2253

Amar-Costesec A., Dublet B. \& Beaufay $H$. (1989) Translocation and proteolytic processing of nascent polypeptide chains : two functions associated with the ribosomal domain of the endoplasmic reticulum. Biol. Cell 65, 99-108

Arias I.M., Doyle D. \& Schimke R.T. (1969) Studies on the synthesis and degradation of 
proteins of the endoplasmic reticulum of rat liver. J. Biol. Chem. 244, 3303-3315

Bar-Nun S., Kreibich G., Adesnik M., Alterman L., Negishi M. \& Sabatini D.D. (1980) Synthesis and insertion of cytochrome P-450 into endoplasmic reticulum membranes. Proc. Natl Acad. Sci. USA 77, 965-969

Beaufay H. \& Amar-Costesec A. (1976) Cell fractionation techniques. In : Methods in Membrane Biology (Korn E.D., ed.). Plenum Press, New York, London, pp. 1-100

Beaufay H., Amar-Costesec A., ThinesSempoux D., Wibo M., Robbi M. \& Berthet J. (1974) Analytical study of microsomes and isolated subcellular membranes from rat liver. III. Subfractionation of the microsomal fraction by isopycnic and differential centrifugation in density gradients. J. Cell Biol. 61, 213-231

Beaufay H., Amar-Costesec A. \& De Duve C. (1981) The topology of enzymes in microsomes from liver. In : Mitochondria and Microsomes (Lee C.P., Schatz G. \& Dallner G., eds). Addison-Wesley Publishing Company, Reading (Mass.), pp. 629-653

Beaufay H., Ravoet A.M., Godelaine D. \& AmarCostesec A. (1985) Subcellular membrane topology of glycosyltransferases involved in $\mathrm{N}$ glycosylation of proteins. In : Aspects Cellulaires et Pathologiques du Métabolisme des Glycoconjugués - Cellular and Pathological Aspects of Glycoconjugate Metabolism (Dreyfus H., Massarelli R., Freysz L. \& Rebel G., eds). Colloque INSERM/CNRS 1984, Les Editions INSERM 1985, 47-66

Bell R.M. \& Coleman R.A. (1980) Enzymes of glycerolipid synthesis in eukaryotes. Ann. Rev. Biochem. 49, 459-487

Blobel G. \& Potter V.R. (1967) Studies on free and membrane-bound ribosomes in rat liver. I. Distribution as related to total cellular RNA. J. Mol. Biol. 26, 279-292

Blouin A., Bolender R.P. \& Weibel E.R. (1977) Distribution of organelles and membranes between hepatocytes and nonhepatocytes in the rat liver parenchyma. A stereological study. J. Cell Biol. 72, 441-455

Bock K.W., Siekevitz P. \& Palade G.E. (1971) Localization and turnover studies of membrane nicotinamide adenine dinucleotide glycohydrolase in rat liver. J. Biol. Chem. 246, 188-195
Bolender R.P. \& Weibel E.R. (1973) A morphometric study of the removal of phenobarbitalinduced membranes from hepatocytes after cessation of treatment. J. Cell Biol. 56, 746-761

Bolender R.P., Paumgartner D., Losa G., Muellener D. \& Weibel E.R. (1978) Integrated stereological and biochemical studies on hepatocytic membranes. I. Membrane recoveries in subcellular fractions. J. Cell Biol. 77, 565-583

Borgese N. \& Gaetani S. (1980) Site of synthesis of NADH-cytochrome $b_{5}$ reductase, an integral membrane protein, in rat liver cells. FEBS Lett. 112, 216-220

Borgese N., Pietrini G. \& Meldolesi J. (1980) Localization and biosynthesis of NADHcytochrome $b_{5}$ reductase, an integral membrane protein, in rat liver cells. III. Evidence for the independent insertion and turnover of the enzyme in various subcellular compartments. J. Cell Biol. 86, 38-45

Chesterton C.J. (1968) Distribution of cholesterol precursors and other lipids among rat liver intracellular structures. Evidence for the endoplasmic reticulum as the site of cholesterol and cholesterol ester synthesis. J. Biol. Chem. 243, 1147-115t

Claude A. (1938) A fraction from normal chick embryo similar to the tumor producing fraction of chicken tumor I. Proc. Soc. Exp. Biol. Med. 39, 398-303

Crimaudo C., Hortsch M., Gausepohl H. \& Meyer D.I. (1987) Human ribophorins I and If : the primary structure and membrane topology of two highly conserved rough endoplasmic reticulum-specific glycoproteins. EMBO J. 6, 75-82

Dailey H.A. \& Strittmatter P. (1981) The role of $\mathrm{COOH}$-terminal anionic residues in binding cytochrome $b_{5}$ to phospholipid vesicles and biological membranes. J. Biol. Chem. 256, 1677-1680

Dallner G. \& Ernster L. (1968) Subfractionation and composition of microsomal membranes : a review. J. Histochem. Cytochem. 16, 611-632

Dallner G., Orrenius S. \& Bergstrand A. (1963) Isolation and properties of rough and smooth vesicles from rat liver. J. Cell Biol. 16, 426-430

Dawidowicz E.A. (1987) Dynamics of membrane lipid metabolism and turnover. Ann. Rev. Biochem. 56, 43-61

Depierre J.W. \& Dallner G. (1975) Structural aspects of the membrane of the endoplasmic reticulum. Biochim. Biophys. Acta 415, 411-472 
Edwards P.A. \& Gould R.G. (1972) Turnover rate of hepatic 3-hydroxy-3-methylglutaryl coenzyme A reductase as determined by use of cycloheximide. J. Biol. Chem. 247, 1520-1524

Estabrook R.W., Masters B.S.S., Capdevila J., Prough R.A., Werringloer J. \& Peterson J.A. (1981) Cytochromes and flavoproteins of the microsomal membrane : past hypotheses and current concepts regarding structure-function relationships. In : Mitochondria and Microsomes (Lee C.P., Schatz G., Dallner G., eds). AddisonWesley Publishing Company, Reading (Mass.), pp. 683-705

Evans E.A., Gilmore R. \& Blobel G. (1986) Purification of microsomal signal peptidase as a complex. Proc. Natl Acad. Sci. USA 83, 581585

Falany C.N., McQuiddy P. \& Kasper C.B. (1987) Structure and organization of the microsomal xenobiotic epoxide hydrolase gene. J. Biol. Chem. 262, 5924-5930

Fujii-Kuriyama Y., Mikawa R., Tashiro Y., Sakai M. \& Maramatsu M. (1978) Seikagaku 50, 870 (en japonais)

Fujii-Kuriyama Y., Negishi M., Mikawa R. \& Tashiro $Y$. (1979) Biosynthesis of cytochrome P450 on membrane-bound ribosomes and the subsequent incorporation into rough and smooth microsomes in rat hepatocytes. J. Cell Biol. 81, 510-519

Fujii-Kuriyama Y., Mizukami Y., Kawajiri K., Sogawa K. \& Muramatsu M. (1982) Primary structure of cytochrome P-450 : coding nucleotide sequence of phenobarbital-inducible cytochrome P-450 cDNA from rat liver. Proc. Natl Acad. Sci. USA 79, 2793-2797

Gellhorn A. \& Benjamin W. (1966) The effect of insulin on monounsaturated fatty acid synthesis in diabetic rats. The stability of the informational RNA and of the enzyme system concerned with fatty acid desaturation. Biochim. Biophys. Acta 116, 460-466

Gielen J., Van Cantfort J., Robaye B. \& Renson J. (1975) Rat-liver cholesterol $7 \alpha$-hydroxylase.

3. New results about its circadian rhythm. Eur. J. Biochem. 55, 41-48

Gilmore R., Blobel G. \& Walter P. (1982) Protein translocation across the endoplasmic reticulum. I. Detection in the microsomal membrane of a receptor for the signal recognition particle. J. Cell Biol. 95, 463-469
Gonzales F.J. \& Kasper C.B. (1980a) Phenobarbital induction of NADPH-cytochrome $c$ (P450) oxidoreductase messenger ribonucleic acid. Biochemistry 19, 1790-1796

Gonzales F.J. \& Kasper C.B. (1980b) In vitro translation of epoxide hydratase messenger RNA. Biochem. Biophys. Res. Commun. 93, 1254-1258

Gonzales F.J., Nebert D.W., Hardwick J.P. \& Kasper C.B. (1985) Complete cDNA and protein sequence of a pregnenolone $16 \alpha$-carbonitrileinduced cytochrome $P-450$. A representative of a new gene family. J. Biol. Chem. 260, 74357441

Harano H. \& Omura T. (1977) Biogenesis of endoplasmic reticulum membrane in rat liver cells. II. Discharge of the nascent peptides of NADPH-cytochrome $c$ reductase and cytochrome $b_{5}$ on the cytoplasmic side of the endoplasmic reticulum membrane. J. Biochem. 82, 1551-1557

Harnik-Ort V., Prakash K., Marcantonio E., Colman D.R., Rosenfeld M.G., Adesnik M., Sabatini D.D. \& Kreibich G. (1987) Isolation and characterization of CDNA clones for rat ribophorin I: complete coding sequence and in vitro synthesis and insertion of the encoded product into endoplasmic reticulum membrane. J. Cell Biol. 104, 855-863

Iyanagi T., Haniu M., Sogawa K., Fujii-Kuriyama Y., Watanabe D., Shively J.E. \& Anan K.F. (1986) Cloning and characterization of CDNA encoding 3-methylcholanthrene inducible rat mRNA for UDP-glucuronosyltransferase. J. Biol. Chem. 261, 15607-15614

Kawajiri K., Ito A. \& Omura T. (1977) Subfractionation of rat liver microsomes by immunoprecipitation and immunoadsorption methods. J. Biochem. 81, 779-789

Kiehn E.D. \& Holland J.J. (1970) Membrane and nonmembrane proteins of mammalian cells. Synthesis, turnover and size distribution. Biochemistry 9, 1716-1728

Kreibich G., Ulrich B.L. \& Sabatini D.D. (1978) Proteins of rough microsomal membranes related to ribosome binding. I. Identification of ribophorins I and II, membrane proteins characteristic of rough microsomes. J. Cell Biol. 77, 464487

Kuriyama Y. (1972) Studies on microsomal nucleoside diphosphatase of rat hepatocytes. Its 
purification, intramembranous localization and turnover. J. Biol. Chem. 247, 2979-2988

Kuriyama Y., Omura T., Siekevitz P. \& Palade G.E. (1969) Effects of phenobarbital on the synthesis and degradation of the protein components of rat liver microsomal membranes. $J$. Biol. Chem. 244, 2017-2026

Lauffer L., Garcia P.D., Harkins R.N., Coussens L., Ulirich A. \& Walter P. (1985) Topology of signal recognition particle receptor in endoplasmic reticulum membrane. Nature 318, 334-338

Liscum L., Finer-Moore J., Stroud R.M., Luskey K.L., Brown M.S. \& Goldstein J.L. (1985) Domain structure of 3-hydroxy-3-methylglutaryl coenzyme A reductase, a glycoprotein of the endoplasmic reticulum. J. Biol. Chem. 260, 522-530

Losa G., Weibel E.R. \& Bolender R.P. (1978) Integrated stereological and biochemical studies on hepatocytic membranes. III. Relative surface of endoplasmic reticulum membranes in microsomal fractions estimated on freeze-fracture preparations. J. Cell Biol. 78, 289-308

Loud A.V. (1968) A quantitative stereological description of the ultrastructure of normal rat liver parenchymal cells. J. Cell Biol. 37, 27-46

Mackenzie P.I. (1986a) Rat liver UDP. glucuronosyitransferase. Sequence and expression of cDNA encoding a phenobarbitalinducible form. J. Biol. Chem. 261, 6119-6125

Mackenzie P.I. (1986a) Rat liver UDP. glucuronosyltransferase. CDNA sequence and expression of a form glucuronidating 3-hydroxyandrogens. J. Biol. Chem. 261, 14112-14117

Mackenzie P.l. (1987) Rat liver UDPglucuronosyltransferase. Identification of cDNAs encoding two enzymes which glucuronidate testosterone, dihydrotestosterone and $\beta$-estradiol. J. Biol. Chem. 262, 9744-9749

Marcantonio E.E., Amar-Costesec A. \& Kreibich G. (1984) Segregation of the polypeptide translocation apparatus to regions of the endoplasmic reticulum containing ribophorins and ribosomes. II. Rat liver microsomal subfractions contain equimolar amounts of ribophorins and ribosomes. J. Cell Biol. 99, 2254-2259

Matsuura S., Fujii-Kuriyama $Y$. \& Tashiro $Y$. (1978) Immunoelectron microscope localization of cytochrome P-450 on microsomes and other membrane structures of rat hepatocytes. J. Cell Biol. 78, 503-519

Matsuura S., Fujii-Kuriyama Y. \& Tashiro $Y$. (1979) Quantitative immunoelectron- microscopic analyses of the distribution of cytochrome P-450 molecules on rat liver microsomes. J. Cell Sci. 36, 413-435

Meyer D.I. \& Dobberstein B. (1980) A membrane component essential for vectorial translocation of nascent proteins across the endoplasmic reticulum : requirements for its extraction and reassociation with the membrane. $J$. Cell Biol. 87, 498-502

Mitropoulos K.A., Venkatesan S., Balasubramaniam S. \& Peters T.J. (1978) The submicrosomal localisation of 3-hydroxy-3-methylglutarylcoenzyme-A reductase, cholesterol $7 \alpha-$ hydroxylase and cholesterol in rat liver. Eur. J. Biochem. 82, 419-429

Molnar J., Tetas M. \& Chao H. (1969) Subcellular site of glycoprotein synthesis in liver. Biochem. Biophys. Res. Commun. 37, 684-690

Morimoto T., Matsuura S., Sasaki S., Tashiro Y. \& Omura T. (1976) Immunochemical and immunoelectron microscope studies on localization of NADPH-cytochrome $c$ reductase on rat liver microsomes. J. Cell Biol. 68, 189-201

Negishi M., Fujii-Kuriyama Y., Tashiro Y. \& Imai $Y$. (1976) Site of biosynthesis of cytochrome $P$ 450 in hepatocytes of phenobarbital treated rats. Biochem. Biophys. Res. Commun. 71, 11531160

Ojakian G.K., Kreibich G. \& Sabatini D.D. (1977) Mobility of ribosomes bound to microsomal membranes. A freeze-etch and thin-section electron microscope study of the structure and fluidity of the rough endoplasmic reticulum. J. Cell Biol. 72, 530-551

Okada Y. \& Omura T. (1978) Synthesis and turnover of microsomal and mitochondrial $\mathrm{NADH}$-cytochrome $b_{5}$ reductases in rat liver. $J$. Biochem. 83, 1039-1048

Okada Y., Sabatini D.D. \& Kreibich G. (1979) Site of synthesis of rat liver cytochrome $b_{5}$ and NADPH-cytochrome P-450 reductase. J. Cell Biol. 83, 437a

Okada Y., Frey A.B., Guenthner T.M., Oesch F., Sabatini D.D. \& Kreibich G. (1982) Studies on the biosynthesis of microsomal membrane proteins. Site of synthesis and mode of insertion of cytochrome $b_{5}$, cytochrome $b_{5}$ reductase, cytochrome $\mathrm{P}-450$ reductase and epoxide hydrolase. Eur. J. Biochem. 122, 393-402

Omura T., Siekevitz P. \& Palade G.E. (1967) Turnover of constituents of the endoplasmic re- 
ticulum membranes of rat hepatocytes. J. Biol. Chem. 242, 2389-2396

Oshino N. \& Sato R. (1972) The dietary control of the microsomal stearyl CoA desaturation enzyme system in rat liver. Arch. Biochem. Biophys. 149, 369-377

Ozols J., Korza G., Heinemann F.S., Hediger M.A. \& Strittmatter P. (1985) Complete amino acid sequence of steer liver microsomal $\mathrm{NADH}$ cytochrome $b_{5}$ reductase. J. Biol. Chem. 260, 11953-11961

Palade G.E. (1956) The endoplasmic reticulum. J. Biophys. Biochem. Cytol. 2, 85-98

Palade G.E. \& Siekevitz P. (1956) Pancreatic microsomes. An integrated morphological and biochemical study. J. Biophys. Biochem. Cytol. 2, 671-690

Porter T.D. \& Kasper C.B. (1985) Coding nucleotide sequence of rat NADPH-cytochrome P450 oxidoreductase cDNA and identification of flavin-binding domains. Proc. Natt Acad. Sci. USA 82, 973-977

Porter T.D., Beck T.W. \& Kasper C.B. (1986) Complementary DNA and amino acid sequence of rat liver microsomal, xenobiotic epoxide hydrolase. Arch. Biochem. Biophys. 248, 121-129

Ravoet A.M., Amar-Costesec A., Godelaine D. \& Beaufay H. (1981) Quantitative assay and subcellular distribution of enzymes acting on dolichyl phosphate in rat liver. J. Cell Biol. 91, 679-688

Reinhart M.P., Billheimer J.T., Faust J.R. \& Gaylor J.L. (1987) Subcellular localization of the enzymes of cholesterol biosynthesis and metabolism in rat liver. J. Biol. Chem. 262, 9649-9655

Robbi M. \& Beaufay H. (1983) Purification and characterization of various esterases from rat liver. Eur. J. Biochem. 137, 293-301

Robbi M. \& Beaufay H. (1986) Biosynthesis of rat-liver pl-5.0 esterases in cell-free systems and in cultured hepatocytes. Eur. J. Biochem. 158, 187-194

Robbi M. \& Beaufay H. (1987) Biosynthesis of rat liver pl-6.1 esterase, a carboxylesterase of the cisternal space of the endoplasmic reticulum. Biochem. J. 248, 545-550

Rohr H.P., Oberholzer M., Bartsch C. \& Keller M. (1976) Morphometry in experimental patholo- gy (methods, baseline data and application). Int. Rev. Exp. Pathol. 15, 233-325

Rosenfeld M.G., Marcantonio E.E., Hakimi J., Ort V.M., Atkinson P.H., Sabatini D. \& Kreibich G. (1984) Biosynthesis and processing of ribophorins in the endoplasmic reticulum. J. Cell Biol. 99, 1076-1082

Rothschild J. (1963) The isolation of microsomal membranes. Biochem. Soc. Symp. 22, 4-31

Tajima S., Lauffer L., Rath V.L. \& Walter P. (1986) The signal recognition particle receptor is a complex that contains two distinct polypeptide chains. J. Cell Biol. 103, 1167-1178

Thiede M.A. \& Strittmatter P. (1985) The induction and characterization of rat liver stearyl-CoA desaturase mRNA. J. Biol. Chem. 260, 1145914463

Trzaskos J.M., Bowen W.D., Fischer G.J., Billheimer J.T. \& Gaylor J.L. (1982) Microsomal enzymes of cholesterol biosynthesis from lanosterol : a progress report. Lipids 17, 250-256

Van Den Eynde B., Beaufay H., Godelaine D. \& Amar-Costesec A. (1985) Assay and topology of UDP-acetylglucosaminedolichylpyrophosphorylacetylglucosamine - acetylglucosaminyl transferase in rat liver. Arch. Internat. Physiol. Biochim. 93, B117-B118

Venkatesan S., Mitropoulos K.A., Balasubramaniam S. \& Peters T.J. (1980) Biochemical evidence for the heterogeneity of membranes from rat liver endoplasmic reticulum. Studies on the localization of acyl-CoA:cholesterol acyltransferase. Eur. J. Cell Biol. 21, 167-174

Weibel E.R., Staubli W., Gnagi H.R. \& Hess F.W. (1969) Correlated morphometric and biochemical studies on the liver cell. I. Morphometric model, stereologic methods and normal morphometric data for rat liver. J. Cell Biol. 42, 6891

Wibo M., Amar-Costesec A., Berthet J. \& Beaufay $H$. (1971) Electron microscope examination of subcellular fractions. III. Quantitative analysis of the microsomal fraction isolated from rat liver. J. Cell Biol. 51, 52-71

Yang C.S. (1977) The organization and interaction of monoxygenase enzymes in the microsomal membrane. Life Sci. 21, 1047-1058 